\title{
Structural and Transport Properties of Li/S Battery Electrolytes: Role of the Polysulfide Species
}

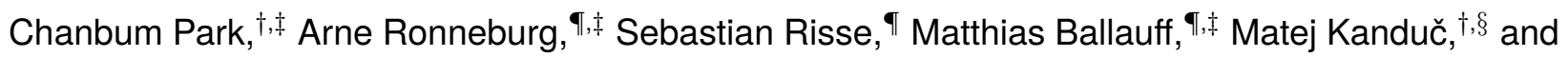 \\ Joachim Dzubiella*,\|,†
}

$\nmid$ Research Group for Simulations of Energy Materials, Helmholtz-Zentrum Berlin für Materialien und Energie, Hahn-Meitner-Platz 1, D-14109 Berlin, Germany

$\ddagger$ Institut für Physik, Humboldt-Universität zu Berlin, Newtonstr. 15, D-12489 Berlin, Germany

ๆInstitut für Weiche Materie und Funktionale Materialien, Helmholtz-Zentrum Berlin für Materialien und Energie, Hahn-Meitner-Platz 1, D-14109 Berlin, Germany

§Jožef Stefan Institute, Jamova 39, SI-1000 Ljubljana, Slovenia

||Applied Theoretical Physics - Computational Physics, Physikalisches Institut, Albert-Ludwigs-Universität Freiburg, Hermann-Herder Strasse 3, D-79104 Freiburg, Germany

E-mail: joachim.dzubiella@physik.uni-freiburg.de

\begin{abstract}
Lithium-sulfur $(\mathrm{Li} / \mathrm{S})$ batteries are regarded as one of the most promising energy storage devices beyond lithium-ion batteries because of their high energy density of $2600 \mathrm{~W} \mathrm{~h} \mathrm{~kg}^{-1}$ and an affordable cost of sulfur. Meanwhile, some challenges inherent to $\mathrm{Li} / \mathrm{S}$ batteries remain to be tackled, for instance, the polysulfide (PS) shuttle effect, the irreversible solidification of $\mathrm{Li}_{2} \mathrm{~S}$, and the volume expansion of the cathode material during discharge. On the molecular level, these issues originate from the structural and solubility behavior of the PS species in bulk and in the electrode confinement. In this study, we use classical molecular dynamics (MD) simulations to develop a working model for PS of different chain lengths in applied electrolyte solutions of lithium bistriflimide (LiTFSI) in 1,2-dimethoxyethane (DME) and 1,3-dioxolane (DOL) mixtures. We investigate conductivities, diffusion coefficients, solvation structures, and clustering behavior and verify our simulation model with experimental measurements available in literature and newly performed by us. Our results show that diffusion coefficients and conductivities are significantly influenced by the chain length of PS. The conductivity contribution of the short chains, like $\mathrm{S}_{4}{ }^{2-}$, is lower than of longer PS chains, such as $\mathrm{S}_{6}{ }^{2-}$ or $\mathrm{S}_{8}{ }^{2-}$, despite the fact that the diffusion coefficient of $\mathrm{S}_{4}{ }^{2-}$ is higher than for longer PS chains. The low conductivity of $\mathrm{Li}_{2} \mathrm{~S}_{4}$ can be attributed to its low degree of dissociation and even to a formation of large clusters in the solution. It is also found that an addition of $1 \mathrm{M}$ LiTFSI into PS solutions considerably reduces the clustering behavior. Our simulation model enables future systematic studies in various solvating and confining systems for the rational design of Li/S electrolytes.
\end{abstract}

\section{INTRODUCTION}

Lithium-ion batteries are the dominant type of rechargeable batteries currently on the market. Relatively efficient battery life cycle, decent energy and power densities make lithium-ion batteries attractive. Meanwhile, the growing demand for large-scale energy storage systems and electric vehicles motivates the battery community to seek new solutions with higher energy density and lower material costs. One of the candidates are lithium-sulfur $(\mathrm{Li} / \mathrm{S})$ batteries owing to a low cost of sulfur, high energy density of $2600 \mathrm{~W} \mathrm{~h} \mathrm{~kg}^{-1}$, and high theoretical specific capacity of $1675 \mathrm{~A} \mathrm{~h} \mathrm{~kg}^{-1}$.12

In spite of these attractive advantages, a practical $\mathrm{Li} / \mathrm{S}$ battery performance has not been realized. Unlike 
lithium-ion batteries, where intercalation of $\mathrm{Li}^{+}$provides the major dynamics, elemental sulfur $S_{8}$ in the cathode chemically reacts with $\mathrm{Li}^{+} .314$ During discharge, $\mathrm{Li}^{+}$migrates from the lithium anode through the electrolyte and the separator to the sulfurized cathode. Subsequently, solid sulfur converts into linear chain-like polysulfide (PS) intermediates, whose length disproportionately shrinks upon reduction with lithium until $\mathrm{Li}_{2} \mathrm{~S}$ finally precipitates. 24] During charging, the reverse chemical reaction partially takes place. As a result of a complex cell chemistry, $\mathrm{Li} / \mathrm{S}$ cells have to face two major challenges. First, parasitic side reactions at the lithium electrode can occur that originate from irreversible re-

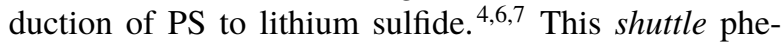
nomenon, originating from the migration of PS from the cathode materials toward the anode, is one source of capacity fading. Second, during discharge $\mathrm{Li}_{2} \mathrm{~S}_{2}$ increase in volume by $80 \%$, which can lead to mechanical stress in the cathode material and loss of contact to the conductive host matrix. $\frac{1899}{18}$ These adverse phenomena are the major bottlenecks of the Li/S battery development. As indicated by recent work, $\frac{210}{20}$ extensive efforts have been devoted into minimizing the shuttling of the PS by confining them into meso- or micro-porous materials. $11-15$ Because the soluble intermediate PS species cause the shuttling, the reaction mechanism pathway can also be altered by choices of the solvent and ions and thus changing the stability of the intermediate species, the charge/discharge rate, etc. $\frac{4[15-20}{20}$ Therefore, more fundamental and systematical studies on the structural and dissolution properties of PS in various applied solvents are in urgent need.

The most frequently formed intermediates during the battery cycling process are $\mathrm{S}_{x}{ }^{2-}$ (with $\left.x=4, \ldots, 8\right)$. These PS ionic chains in the electrolyte can merge together with $\mathrm{Li}^{+}$into clusters, such as $\left(\mathrm{Li}_{2} \mathrm{~S}_{x}\right)_{n}$ (with $\left.n \geq 1\right) .{ }^{21}$ The clusters of $\mathrm{Li}_{2} \mathrm{~S}_{4}$ are believed to be the last polysulfide intermediates before the formation of $\mathrm{Li}_{2} \mathrm{~S}_{2} / \mathrm{Li}_{2} \mathrm{~S}$ insoluble aggregates during the discharge process. ${ }^{22}$ In recent years, various quantum chemistry calculations and classical MD models demonstrated the poor solubility and thus the tendency for cluster formation of shorter PS species. 21.29

The solubility of PS, the size distribution of the PS clusters and their morphologies depend on the properties of the solvent and added electrolyte, 30 current density, and temperature $\frac{34}{} \mathrm{~A}$ recent study by Andersen et al ${ }^{35}$ investigated PS clustering using classical MD simulations, DFT calculations and experiments. Another classical MD simulations by Rajput et al. ${ }^{28}$ suggested that introducing TFSI $^{-}$ions, which compete with PS for
$\mathrm{Li}^{+}$, weakens the PS $-\mathrm{Li}^{+}$clustering networks, resulting in higher solubility of PS. However, the diffusion coefficients in that study differ from those measured by the pulse-field gradient nuclear magnetic resonance (PFGNMR) methods by more than one order of magnitude. 28 The control of clustering may thus represent the critical objective for battery performance. It is thus of utmost importance to obtain deeper understanding into the structural and transport properties of these highly complex, multi-component PS solutions.

Our endeavor of this and a preceding study 36 is to develop an efficient yet accurate force field for purely classical molecular dynamics simulations of $\mathrm{Li} / \mathrm{S}$ battery electrolytes. We have successfully described static and transport properties of the solutions of DME/DOL containing LiTFSI and $\mathrm{LiNO}_{3}$ salts. ${ }^{36}$ In this study, we implement PS components into the previously developed model. 36 For the most relevant PS chain lengths of 4, 6, and 8 sulfur atoms, we investigate clustering behavior of PS, their solvation structure, diffusion, and conductivity properties.

\section{METHODS}

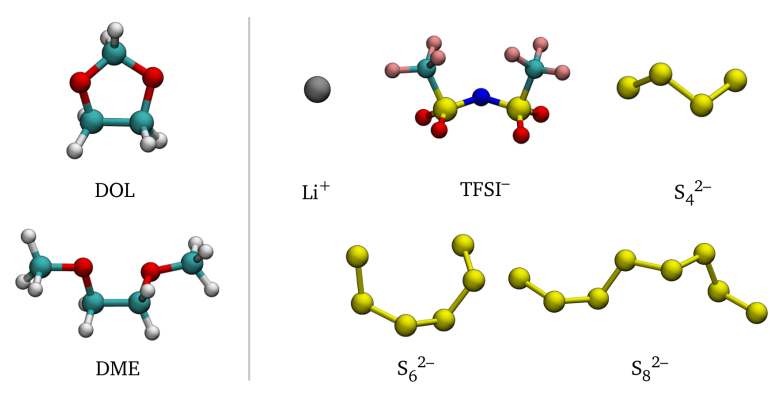

Figure 1: Solvent molecules 1,3-Dioxolane (DOL) and 1,2-Dimethoxyethane (DME) and ions $\mathrm{Li}^{+}$, Bis(trifluoromethanesulfonyl)imide $\left(\mathrm{TFSI}^{-}\right)$, and the polysulfides (PS) with chain lengths $x=4,6$, and 8 considered in this study.

2.1. Model and force fields. The solvent in our atomistic model is a 1:1 molar mixture of 1,2-Dimethoxyethane (DME) and 1,3-Dioxolane (DOL), which contains different amounts of $\mathrm{Li}^{+}$and Bis(trifluoromethanesulfonyl)imide ions $\left(\mathrm{TFSI}^{-}\right.$), as well as three different kinds of PS ions: $\mathrm{S}_{4}{ }^{2-}, \mathrm{S}_{6}{ }^{2-}, \mathrm{S}_{8}{ }^{2-}$ (see Fig. 1).

We employ the OPLS-AA force field ${ }^{37 / 38}$ for DME and DOL, the CL \& $\mathrm{P}$ force field for TFSI ${ }^{-}, 39$ and Dang et al. ${ }^{40}$ for the $\mathrm{Li}^{+}$ion, together with the geometric combination rules for Lennard-Jones (LJ) interactions. The 
Coulomb interactions of ions are treated by the electronic continuum correction (ECC) method, 41 which takes the electronic polarizability into account implicitly. In this approach, formal ionic charges $q_{i}$ in the interaction Hamiltonian are replaced by effective, rescaled values $q_{i}^{\mathrm{eff}}$,

$$
q_{i}^{\mathrm{eff}}=\frac{q_{i}}{\sqrt{\epsilon_{\infty}}}
$$

where $\epsilon_{\infty}=1.93$ is the high-frequency dielectric permittivity of the bare solvent. ${ }^{\sqrt{36}}$ This approach turned out to be very successful for many structural and dynamic properties of $\mathrm{LiTFSI} / \mathrm{LiNO}_{3}$ salts in DME/DOL solutions $\frac{36}{}$ and is applied in this study also to the PS ions. We devote special attention to the parametrization of the PS ions. The bonded parameters for PS ions are taken from the recent work by Rajput et al. ${ }^{28}$ In order to obtain the partial charges, we performed quantum mechanical calculations using the electrostatic potential surface method implemented in the GAUSSIAN09 package 48 with the B3LYP functional at the aug-cc-pvdz basis set level. The resulting (unscaled) and the corresponding effective (rescaled via Eq. 1) partial charges of sulfur atoms in PS are summarized in Table 1.

Table 1: Formal $\left(q_{i}\right)$ and effective ( $q_{i}^{\text {eff }}$, computed via Eq. 1) partial charges of terminal and internal $S$ atoms in the PS chains. $e$ is the elementary charge.

\begin{tabular}{|l|c|c|c|c|}
\hline \hline & \multicolumn{2}{|c|}{ Terminal S } & \multicolumn{2}{c|}{ Internal S } \\
\hline & $q_{i} / e$ & $q_{i}^{\text {eff }} / e$ & $q_{i} / e$ & $q_{i}^{\text {eff }} / e$ \\
\hline $\mathrm{S}_{4}{ }^{2-}$ & -0.7702 & -0.5546 & -0.2298 & -0.1655 \\
$\mathrm{~S}_{6}{ }^{2-}$ & -0.6537 & -0.4707 & -0.1731 & -0.1247 \\
$\mathrm{~S}_{8}{ }^{2-}$ & -0.6223 & -0.4481 & -0.1259 & -0.0907 \\
\hline \hline
\end{tabular}

Different LJ parameters for $\mathrm{S}$ atoms have been tested using various force fields: OPLS-AA, $\stackrel{37}{ }$ AMBER99, 49 CHARMM,$\stackrel{50}{ }$ ENCAD $^{51}$ ECEPP, ${ }^{52}$ UFF ${ }^{53}$ and DREI$\mathrm{DING}^{54}$ [see Table S1 in the Electronic Supplementary Information (ESI)]. As it turns out, the LJ interaction size $\sigma_{\mathrm{S}-\mathrm{Li}^{+}}$between a terminal $\mathrm{S}$ atom and $\mathrm{Li}^{+}$is a critical parameter that determines the degree of aggregation and clustering propensities, as well as conductivity of PS ions. Therefore, we tuned the LJ parameter manually to $\sigma_{\mathrm{S}-\mathrm{Li}^{+}}=0.275 \mathrm{~nm}$ in order to reproduce experimental conductivity values. The procedure and the details are provided in ESI (Figs. S1 and S2).

2.2. Simulation details and protocols. All-atom MD simulations are carried out with the GROMACS 5.1 sim- ulation package. ${ }^{[55}$ Initial simulation structures of ions in the solvent are constructed with the PACKMOL package. 56 The molecules are randomly inserted into the simulation box and undergo an energy minimization, followed by a simulated annealing from $440 \mathrm{~K}$ down to $298 \mathrm{~K}$ within a time interval of $3 \mathrm{~ns}$ in the $N V T$ ensemble. The production simulations are carried out in the $N p T$ ensemble at a constant pressure of 1 bar and temperature $298 \mathrm{~K}$. The pressure is controlled by the Parrinello-Rahman barostat with the time constant of 2 ps, whereas the temperature is controlled by the Berendsen thermostat with the time constant $0.1 \mathrm{ps}$. ${ }^{[5]}$ The LJ potentials are truncated at $1.3 \mathrm{~nm}$. The Particle-MeshEwald (PME) method with a Fourier spacing of 0.12 $\mathrm{nm}$ and a $1 \mathrm{~nm}$ real-space cut-off are used in calculating electrostatic interactions. The LINCS algorithm is used for all bond constraints.

\subsection{Analysis.}

\subsubsection{Dielectric constant}

The non-electronic part of the static dielectric constant in the simulations, $\epsilon_{\mathrm{MD}}$, is evaluated from the fluctuations of the system's dipole moment (excluding ionic monopoles) $\mathbf{M}_{\mathrm{MD}}$ (using rescaled charges),

$$
\epsilon_{\mathrm{MD}}=1+\frac{1}{3 V \epsilon_{0} k_{\mathrm{B}} T}\left(\left\langle\mathbf{M}_{\mathrm{MD}}^{2}\right\rangle-\left\langle\mathbf{M}_{\mathrm{MD}}\right\rangle^{2}\right),
$$

where $V, k_{\mathrm{B}}, T$ stand for the volume of the simulation box, the Boltzmann constant, and the absolute temperature, respectively. Since we are employing the ECC approach, the total effective dielectric constant is

$$
\epsilon=\epsilon_{\infty} \epsilon_{\mathrm{MD}}
$$

More details can be found in Ref. 36

\subsubsection{Diffusion coefficients}

The self-diffusion coefficients of ions and molecules are computed via the mean square displacement relation,

$$
D_{\mathrm{MD}}=\lim _{\Delta t \rightarrow \infty} \frac{\left\langle r^{2}(\Delta t)\right\rangle}{6 \Delta t} .
$$

In addition, the finite-size effects of the simulation box are corrected by extrapolating measured $D_{\mathrm{MD}}$ values at different box sizes $L$ to $L \rightarrow \infty$ (see Fig. S3 in ESI). 
2.4. Conductivity. Conductivities of the solutions in our simulations are computed from Ohm's law

$$
J=\kappa E,
$$

where $E$ is an applied electric field and $J=\sum_{i} J_{i}=$ $\sum_{i} \kappa_{i} E$ is the resulting total current density. This relation defines partial ionic conductivities $\kappa_{i}$ for each individual ionic species. The conductivities $\kappa_{i}$ are evaluated from the linear slope of $J$ versus $E$ in the linear-response regime (see Fig. S1 in ESI).

\subsubsection{Clustering}

We define a cluster of PS ions as a group of those PS ions whose at least one of the terminal $\mathrm{S}$ atoms is separated from a terminal $\mathrm{S}$ atom of any other PS ion in the cluster by less than $r_{0}=0.53 \mathrm{~nm}$. The cutoff value $r_{0}$ is chosen as the first minimum after the main peak of the radial distribution function (RDF) of terminal $\mathrm{S}$ atoms and thus corresponds to the distance between two terminal $\mathrm{S}$ atoms that have a bridging $\mathrm{Li}^{+}$ion in between (see Fig. S4 in ESI). By ensemble averaging of clusters in the simulations, we obtain an equilibrium cluster size distribution $P(N)$, where $N$ is the number of PS ions in a cluster. The statistical uncertainties are calculated with the block averaging procedure.

\subsubsection{Coordination number and dissociated $\mathrm{Li}^{+}$ion}

The coordination number $N_{i, j}$ of the molecule of type $i$ that is surrounded by molecules of type $j$ is computed from the RDF as $\underline{60}$

$$
N_{i, j}=4 \pi c_{j} \int_{0}^{R_{\mathrm{M}}} g_{i j}(r) r^{2} \mathrm{~d} r,
$$

where $R_{\mathrm{M}}$ is the distance of the first minimum after the first peak in the RDF and $c_{j}$ is the bulk concentration of molecules of the type $j$.

2.5. Experimental Methods. Electrolyte mixtures were prepared by adding the corresponding stoichiometric ratio of lithium sulfide $\left(\mathrm{Li}_{2} \mathrm{~S}\right)$ and elemental sulfur $\left(\mathrm{S}_{8}\right)$ to a mixture of DME and DOL (1:1 by mole). The uncertainty on the composition of the electrolyte is less than 5\%. Although the various disproportion reactions of lithium polysulfides in solution are known, it is assumed that the majority of PS appears in the length as prepared by the intended stoichiometric ratio, as shown by Barchasz et al. using high-performance liquid chromatography (HPLC). ${ }^{61}$ The gravimetric density of the
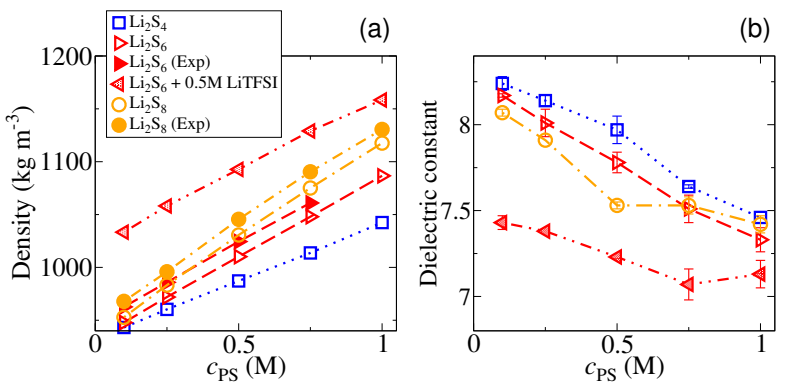

Figure 2: (a) Density and (b) dielectric constant as a function of the polysulfide concentration in DME/DOL (1:1) solvent in MD simulations. The experimental measurements for $\mathrm{Li}_{2} \mathrm{~S}_{6}$ and $\mathrm{Li}_{2} \mathrm{~S}_{8}$ are depicted by solid triangles and circles in panel (a).

electrolyte mixtures were measured at $18{ }^{\circ} \mathrm{C}$ and 1 bar using a chempro/PAAR DMA 602 density meter. The average value of ten measurements of the natural frequency of a glass tube filled with the solution was taken to calculate the density. Millipore water and air served as reference for this calculation. The error on the values is $2 \times 10^{-5}$. The viscosity was determined using a Capillary Viscometer (SI Analytics 50101/0a) and a laboratory stop-watch. The measurement were performed in an argon filled glovebox at $25^{\circ} \mathrm{C}$. The error of the measurement varies from $0.07 \%$ to $1 \%$. The conductivity was evaluated by performing an impedance spectroscopy in the frequency range of $100 \mathrm{mHz}-1 \mathrm{MHz}$ with $5 \mathrm{mV}$ RMS voltage signal and 20 points per decade. Three different DC voltages $(0,1$, and $1.5 \mathrm{~V})$ were used. A GAMRY interface 1000 potentiostat was used. The cell was a standard CR 2032 coin cell. Two stainless steel plates were used as electrodes. A PTFE-ring (thickness of $0.27 \mathrm{~mm}$, diameter of $10 \mathrm{~mm}$ ) was used to adjust the distance between the two electrodes. The conductivity was determined by the intersection of the impedance with the $x$-axis at high frequencies in a Nyquist-plot.

\section{RESULTS}

3.1. Density and dielectric constant. We start our analysis by calculating the density of various PS in DME/DOL solutions, results of which are plotted in Fig. 2a. Universally, the density is an increasing function of the $\mathrm{Li}_{2} \mathrm{~S}_{x}$ concentration for all PS types. This rise and the magnitude is in good accordance with our experimental measurements for $\mathrm{Li}_{2} \mathrm{~S}_{6}$ and $\mathrm{Li}_{2} \mathrm{~S}_{8}$ (solid triangles and circles, respectively, in Fig. 22). Note that in our simulations we only consider one-component (i.e., polydisperse) PS solutions. In reality, however, the monodis- 
persity cannot be reached due to the disproportionation reactions of PS, but the majority of the PS should appear in the length as prepared.

The dielectric constant as a function of $\mathrm{Li}_{2} \mathrm{~S}_{x}$ concentration is shown in Fig. 2p. It decreases with ion concentration, as is also the case in other electrolytes. ${ }^{62] 65}$ This decrement of the dielectric constant is caused by a local dielectric saturation. Namely, solvent molecules tend to strongly orient and anchor around an ion and do not contribute to the dielectric constant. As more ions are present in the solution, larger fraction of solvent molecules are part of the solvation shells of the ions and thus larger is the local dielectric saturation. The dielectric decrement is slightly larger for longer PS chains. The addition of $0.5 \mathrm{M}$ LiTFSI into the $\mathrm{Li}_{2} \mathrm{~S}_{6}$ solution decreases the dielectric constant considerably, which can be also explained by the local dielectric saturation due to added ions into the solution.

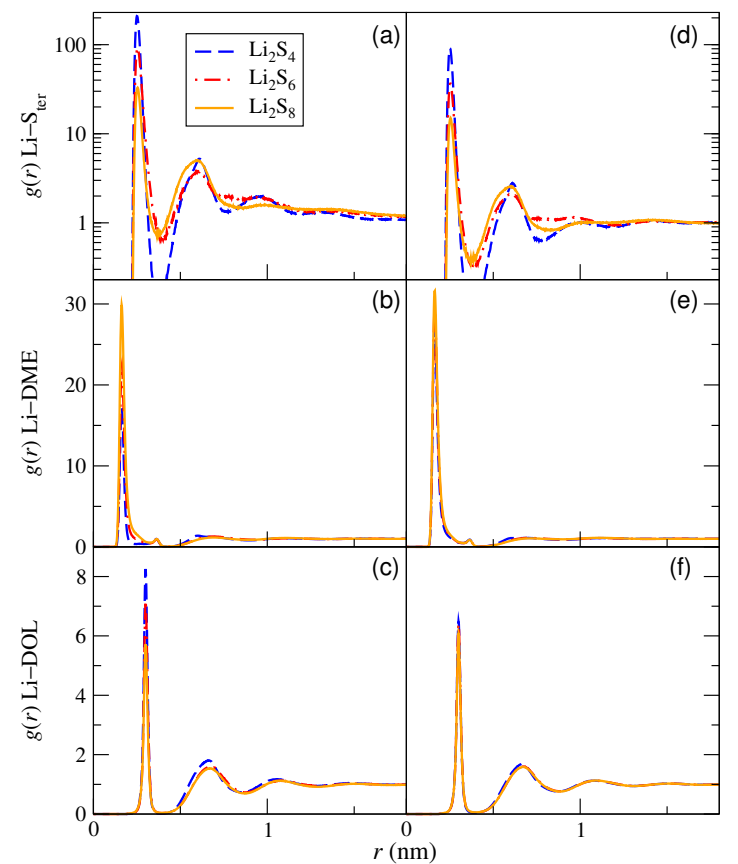

Figure 3: Radial distribution functions (at $0.25 \mathrm{M} \mathrm{Li}_{2} \mathrm{~S}_{x}$ ) between $\mathrm{Li}^{+}$and $(\mathrm{a}, \mathrm{d})$ terminal $\mathrm{S}\left(\mathrm{S}_{\mathrm{ter}}\right)$ in $\mathrm{S}_{x}{ }^{2-}(x=4,6$, and 8), (b, e) DME and (c, f) DOL in DME:DOL (a-c) and in DME:DOL with 1M LiTFSI (d-f).

3.2. Solvation structure and radial distribution functions (RDFs). In the following, we take a look at the solvation structure of $\mathrm{Li}^{+}$ions. Figure 3 shows RDFs of various molecules and $\mathrm{Li}^{+}$ions in DME:DOL [panels $(\mathrm{a}-\mathrm{c})$ ] and in DME:DOL with 1M LiTFSI [panels (d-f)]. The top two panels (a, d) show the distribution of terminal $S$ atoms $\left(S_{\text {ter }}\right)$ of PSs. The very high first peak in all the cases indicates a strong binding affinity between $\mathrm{Li}^{+}$and PS ions and can be attributed to the electrostatic attraction between $\mathrm{Li}^{+}$and $\mathrm{S}_{x}{ }^{2-}$. Moreover, the height of the peak, and with that the binding strength, are diminishing with the length of the PS ions (when going from $\mathrm{S}_{4}{ }^{2-}$ to $\mathrm{S}_{8}{ }^{2-}$ ). These trends are consistent with a recent classical MD simulation study. $\underline{28}$ This can be explained by stronger charge localization (of the net valency -2 ) at terminal ends of shorter PS ions. ${ }^{26}$ As seen in Table 1 . shorter chains have higher partial charges at the termini, thus facilitating the attraction with $\mathrm{Li}^{+}$. Furthermore, the geometry of the PS chains also plays a role in the solvation shell. Snapshots in Figs. 4a, c, e show that short PS chains (e.g., $\mathrm{S}_{4}{ }^{2-}$ ) are able to tightly wrap around a $\mathrm{Li}^{+}$ion. Conversely, longer PS chains (e.g., $\mathrm{Li}_{2} \mathrm{~S}_{6}$ and $\mathrm{Li}_{2} \mathrm{~S}_{8}$ ) do not pack so tightly around the $\mathrm{Li}^{+}$.

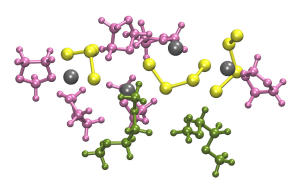

(a) $0.25 \mathrm{M} \mathrm{Li}_{2} \mathrm{~S}_{4}$

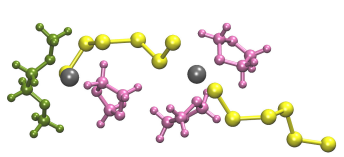

(c) $0.25 \mathrm{M} \mathrm{Li}_{2} \mathrm{~S}_{6}$

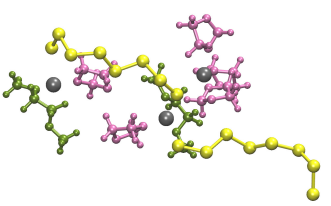

(e) $0.25 \mathrm{M} \mathrm{Li}_{2} \mathrm{~S}_{8}$

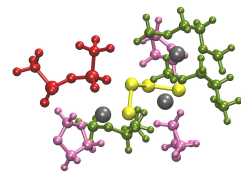

(b) $0.25 \mathrm{M} \mathrm{Li}_{2} \mathrm{~S}_{4}$ and $1 \mathrm{M} \mathrm{LiTFSI}$

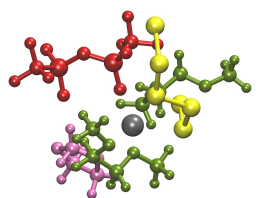

(d) $0.25 \mathrm{M} \mathrm{Li}_{2} \mathrm{~S}_{6}$ and $1 \mathrm{M} \mathrm{LiTFSI}$

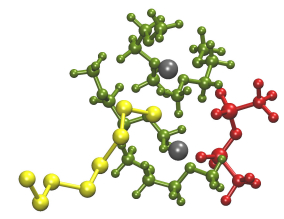

(f) $0.25 \mathrm{M} \mathrm{Li}_{2} \mathrm{~S}_{8}$ and $1 \mathrm{M} \mathrm{LiTFSI}$
Figure 4: Snapshots of $\mathrm{Li}^{+}$solvation shell in DME:DOL and different amounts of ions. Color code: $\mathrm{Li}^{+}$(gray), $\mathrm{S}_{x}{ }^{2-}$ (yellow), FFSI $^{-}$(red), DME (green), DOL (pink).

The different binding strengths result into different com- 
positions of the $\mathrm{Li}^{+}$solvation shell, which can be described by coordination numbers in Fig. 5 Namely, shorter PS chains drive out other molecular species from the first hydration shell of $\mathrm{Li}^{+}$[panels $(\mathrm{a}-\mathrm{d})$ ]. Due to this PS packing in the first solvation shell of $\mathrm{Li}^{+}$, fewer DME or DOL molecules can populate the surrounding of $\mathrm{Li}^{+}$in the presence of $\mathrm{Li}_{2} \mathrm{~S}_{4}$ than in the cases of $\mathrm{Li}_{2} \mathrm{~S}_{6}$ or $\mathrm{Li}_{2} \mathrm{~S}_{8}$ (lower peak in $g_{\mathrm{Li}^{+}-\mathrm{DME}}(r)$ in the case of $\mathrm{Li}_{2} \mathrm{~S}_{4}$ than in $\mathrm{Li}_{2} \mathrm{~S}_{8}$ in Fig. 3p). The DME coordination number of $\mathrm{Li}^{+}$in Fig. 5 increases from $\mathrm{Li}_{2} \mathrm{~S}_{4}$ to $\mathrm{Li}_{2} \mathrm{~S}_{8}$. Ab-initio MD simulations by Kamphaus ${ }^{26}$ also showed similar trends. This densely packed solvation structure by $\mathrm{S}_{4}{ }^{2-}$ gives less chance for $\mathrm{Li}^{+}$in the solvation shell to contact with solvent molecules. It restricts the $\mathrm{Li}^{+}$ exchange between $\mathrm{S}_{4}{ }^{2-}$ and solvent molecules, resulting in lower solubility. $\frac{2666}{616}$

Now we investigate the effects of LiTFSI in the solution. Figure $3 \mathrm{~d}$ shows that the magnitudes of the main peaks in $g_{\mathrm{Li}^{+}-\mathrm{S}_{x}{ }^{2-}}(r)$ decrease after $1 \mathrm{M}$ of LiTFSI is added (cf. panel a), as also consistent with previous studies ${ }^{28}$ (also see $g_{\mathrm{Li}^{+}-\mathrm{TFSI}^{-}}(r)$ in Fig. S5 in ESI). An important insight can be gained from the $\mathrm{Li}^{+}$coordination number around a PS molecule shown in Fig. 5e. Evidently, the number of $\mathrm{Li}^{+}$ions around $\mathrm{S}$ termini does not change upon introducing LiTFSI into the system. This means that LiTFSI does neither weaken the $\mathrm{Li}^{+}-\mathrm{PS}$ bonds nor do additional $\mathrm{Li}^{+}$ions from LiTFSI bind to PS. Thus the decrease in the RDF peaks (3d) and the different solvation shell composition (Fig. 5d) arrive on the expense of the added $\mathrm{Li}^{+}$ions.

3.3. Conductivity. In Fig. 6 we show the ionic conductivity from the MD simulations and experimental measurements (ours, by Safari et al., 67 and by Fan et $\left.a l .{ }^{20}\right)$ for PS concentrations in the range of 0.1-1M. As seen, the experimental trends of the four studied systems $\left(\mathrm{LiS}_{4}, \mathrm{LiS}_{6}, \mathrm{LiS}_{8}\right.$, and $\left.\mathrm{LiS}_{6}+0.5 \mathrm{M} \mathrm{LiTFSI}\right)$ are well captured by the simulations. In cases of $\mathrm{Li}_{2} \mathrm{~S}_{6}$ and $\mathrm{Li}_{2} \mathrm{~S}_{8}$, the conductivities are by a factor of three higher than in experiments. Nevertheless, we regard the results satisfactory as these quantities are extremely sensitive to the molecular interactions and thus prone to large errors, sometimes of more than an order of magnitude. The conductivities from the simulations for the ternary electrolytes of $\mathrm{Li}_{2} \mathrm{~S}_{6}+\mathrm{LiTFSI}$ are also congruent with experiments, capturing even the saturation and the decrease in the conductivity with increasing PS concentration above $0.5 \mathrm{M}$.

Ideal ionic conductivity in the limit of low concentrations

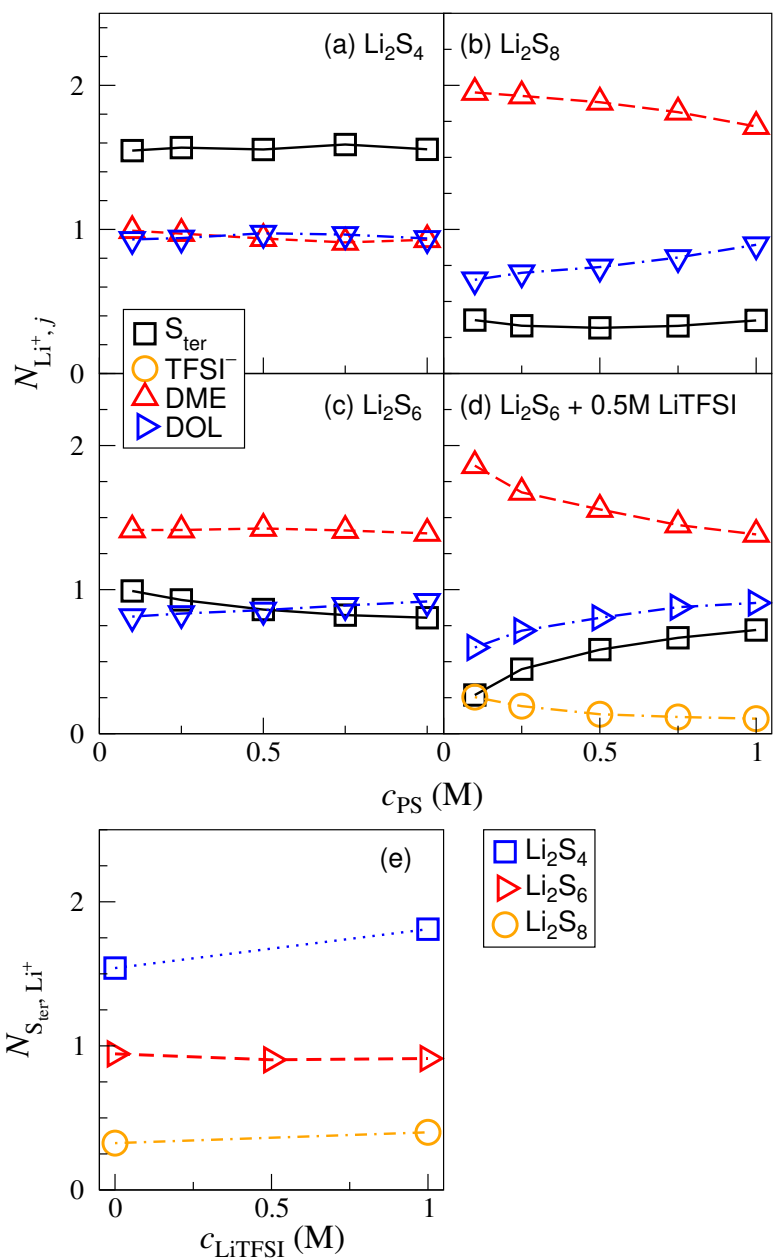

Figure 5: (a-d) Coordination numbers of molecules $j$ around the $\mathrm{Li}^{+}$ion $\left(N_{\mathrm{Li}}+, j\right)$ as a function of the polysulfide concentration (see legend). (e) $\mathrm{Li}^{+}$coordination number around $\mathrm{S}_{\text {ter }}$ $\left(N_{\mathrm{S}_{\mathrm{ter}}, \mathrm{Li}^{+}}\right)$as a function of LiTFSI concentration.

is given by the Nernst-Einstein (NE) equation

$$
\kappa^{\mathrm{id}}=\frac{e^{2}}{k_{\mathrm{B}} T} \sum_{i} z_{i}^{2} c_{i} D_{i}
$$

where $z_{i}$ stands for the ion valency and $c_{i}$ for the ion concentration of species $i$. Using the diffusivities $D_{i}$ obtained from the simulations, we calculate the ideal conductivities in Fig. 6 (triangles). Clearly, the values are an order of magnitude too high, which we attribute to substantial ionic pairing ${ }^{20136}$ Namely, Eq. 7 is a limiting law and thus neglects ion-ion correlations.

In a first-order correction to the ideal conductivity, the correlations can be perturbatively taken into account, 

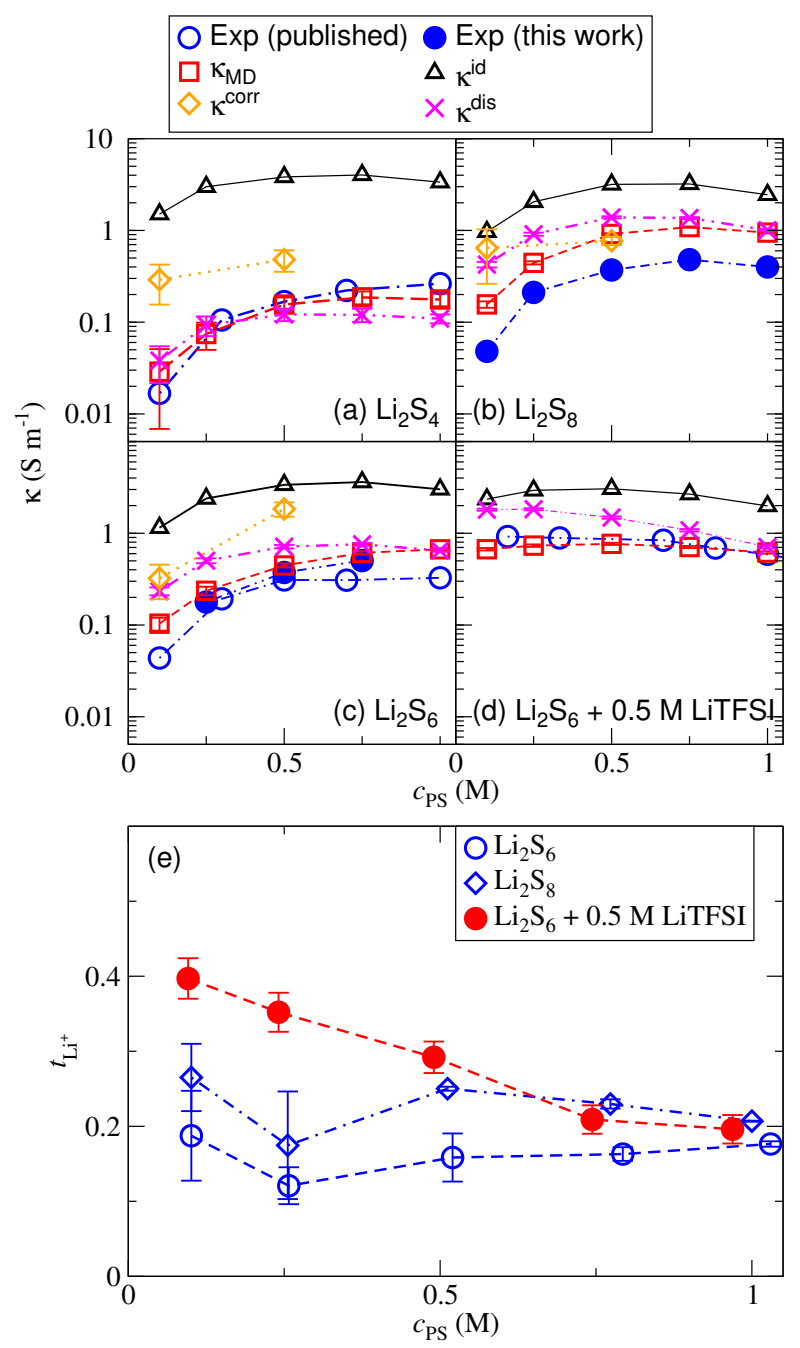

Figure 6: (a-d) Conductivities from MD simulations (squares, triangle left and right), experimental measurements (circles) and the ideal ionic conductivity assuming Eq.7(triangles up) as a function of the polysulfide concentration. (a) $\mathrm{Li}_{2} \mathrm{~S}_{4}$; exp. by Safari et al., 67 (DME:DOL) (b) $\mathrm{Li}_{2} \mathrm{~S}_{8}$; exp. by us (DME:DOL), (c) $\mathrm{Li}_{2} \mathrm{~S}_{6}$; exp. by Safari et al. ${ }^{67}$ (empty circles) and by us (filled circles) (DME:DOL). (d) $\mathrm{Li}_{2} \mathrm{~S}_{6}+0.5 \mathrm{M}$ LiTFSI; exp. by Fan et al. .20 (0.5 M LiTFSI DME:DOL). Diamonds in panels (a), (b) and (c) are $\kappa^{\text {corr }}$ using Eq. 8 Crosses in panels (a), (b), (c) and (d) are $\kappa^{\text {dis }}$ using Eq. 9 (e) Estimated $\mathrm{Li}^{+}$transference number from MD for different $\mathrm{Li}_{2} \mathrm{~S}_{x}$ concentrations (with/without LiTFSI).

which leads to $\underline{68}$

$$
\kappa^{\mathrm{corr}}=\kappa^{\mathrm{id}}+\frac{2 e^{2}}{3 \eta} \sum_{i, j} z_{i} z_{j} c_{i} c_{j} \int_{0}^{\infty} h_{i j}(r) r \mathrm{~d} r
$$

where $\eta$ is the solvent viscosity. Here, the ion-ion correlation effects are expressed via $h_{i j}(r)=g_{i j}(r)-1$. Equation 8 thus constitutes a useful structure-transport relationship, applicable at least for not too dense solutions. Taking $h_{i j}(r)$ from our simulations, we plot the correlation-corrected values $\kappa^{\text {corr }}$ as diamond symbols in Fig. 6 Evidently, the negative effect of ionic pairing is qualitatively captured by the correlation term and the values approach closer the MD results. Among all the contributing ion pairs to the second term in Eq. 8 , the most of contribution comes from the $\mathrm{Li}^{+}-\mathrm{S}_{x}{ }^{2-}$ pair (see $\int_{0}^{\infty} h_{i j}(r) r \mathrm{~d} r$ values in Table $\mathrm{S} 2$ ). This means that the strong binding between the latter two ions is the main culprit for the observed low conductivity. Still, the correlation correction given by Eq. 8 cannot provide a fair quantitative agreement with the measured values and hence we conclude that even at lower concentrations already correlations beyond the pair level are important, e.g., from clustering effects, see further below.

Thus, in the limit of very strong ion pairing, where most of the ions are associated into neutral ion pairs and clusters, we can expect that only the dissociated ions contribute to the conductivity. In this simplified picture, we replace ionic concentrations $c_{i}$ in the NE equation (7) by the concentrations of dissociated ions, $\omega_{i} c_{i}$,

$$
\kappa^{\mathrm{dis}}=\frac{e^{2}}{k_{\mathrm{B}} T} \sum_{i} z_{i}^{2} \omega_{i} c_{i} D_{i}
$$

where $\omega_{i}$ stands for the fraction of dissociated ions of type $i$. For simplicity of our treatment, we assume that only $\mathrm{Li}^{+}$and PS ions are involved in pairing, whereas TFSI $^{-}$ions are completely dissociated, $\omega_{\mathrm{TFSI}^{-}}=1$, as they only weakly bind with $\mathrm{Li}^{+}$. $\mathrm{Li}^{+}$and PS species are subject to the following equilibrium

$$
\mathrm{Li}_{2} \mathrm{~S}_{x} \rightleftharpoons \mathrm{Li}^{+}+\left(\mathrm{LiS}_{x}\right)^{-} .
$$

We assume that further dissociation into $2 \mathrm{Li}^{+}+\mathrm{S}_{x}{ }^{2-}$ is much less probable and we neglect it.

Figure 7 shows the fraction of dissociated $\mathrm{Li}^{+}$in various solutions, defined as the population of those $\mathrm{Li}^{+}$ions that do not have PS in their hydration shells. The dissociation is by far the lowest for $\mathrm{Li}_{2} \mathrm{~S}_{4}$ and higher for $\mathrm{Li}_{2} \mathrm{~S}_{6}$ and $\mathrm{Li}_{2} \mathrm{~S}_{8}$, similar trends are also observed by a recent study.$\underline{35}$ Adding LiTFSI increases the dissociated degree noticeably, which implies that the additional lithium from LiTFSI remains more or less dissociated. In cases without LiTFSI, the effective concentrations of $\mathrm{Li}^{+}$ and $\left(\mathrm{LiS}_{x}\right)^{-}$species are both equal to $2 c_{\mathrm{PS}} \omega_{\mathrm{Li}^{+}}$. Introducing LiTFSI adds equivalent concentrations of $c_{\mathrm{TFSI}^{-}}$ to $\mathrm{Li}^{+}$as well as $\mathrm{TFSI}^{-}$species, since the added $\mathrm{Li}^{+}$are 


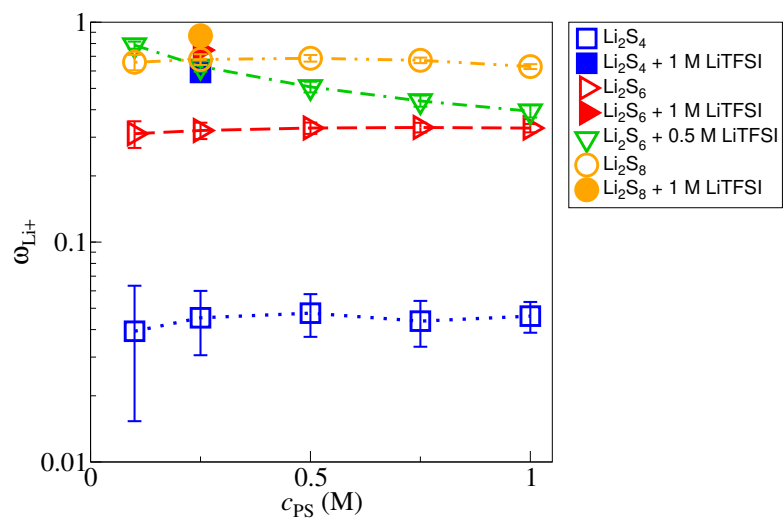

Figure 7: Fraction of dissociated $\mathrm{Li}^{+}$ions as a function of the polysulfide concentration in different solutions. It is defined as the fraction of the $\mathrm{Li}^{+}$population without PS ions in their hydration shells.

all dissociated. Thus, the modified NE equation then reads

$$
\begin{aligned}
\kappa^{\mathrm{dis}}=\frac{e^{2}}{k_{\mathrm{B}} T} & {\left[\left(2 c_{\mathrm{PS}} \omega_{\mathrm{Li}^{+}}+c_{\mathrm{TFSI}^{-}}\right) D_{\mathrm{Li}^{+}}\right.} \\
& \left.+2 c_{\mathrm{PS}_{\mathrm{S}}} \omega_{\mathrm{Li}^{+}} D_{\mathrm{PS}}+c_{\mathrm{TFSI}^{-}} D_{\mathrm{TFSI}^{-}}\right],
\end{aligned}
$$

Note that all the species in this treatment are monovalent, therefore $z_{i}^{2}=1$. In Fig. 6a-d, we plot the conductivities $\kappa^{\text {dis }}$ from Eq. 11 (cross symbols), which in most cases approach much closer to the experimental and MD results than the other two theoretical approaches.

Finally, all three theoretical approaches help us to elucidate the conductivity mechanism of $\mathrm{Li}_{2} \mathrm{~S}_{x}$ solutions. Due to high Li-PS pairing, most of the ion carriers are 'neutralized' and do not contribute to the conductivity. Only the associated fraction acts on the external electric field, which results into an electric current. When LiTFSI salt is added, it contributes mostly dissociated $\mathrm{Li}^{+}$and $\mathrm{TFSI}^{-}$ions and therefore fully contribute to the conductivity, which also explains the almost constant trend in Fig. 6d.

As reported by Zheng et al.,69 a practical sulfur/electrolyte (S/E) ratio (i.e., density of sulfur in electrolyte) with improved cycling stability and Coulombic efficiency are achieved for S/E ratio of $50 \mathrm{~g} \mathrm{~L}^{-1}$. This corresponds to approximately $0.4 \mathrm{M} \mathrm{Li}_{2} \mathrm{~S}_{4}$ in our systems for a complete conversion of all sulfur into $\mathrm{Li}_{2} \mathrm{~S}_{4}$. Above this concentrations, the saturation of the conductivity caused by increasing ionic pairing and viscosity can be one of the limiting factors ${ }^{20}$ for using the high S/E ratio solutions.

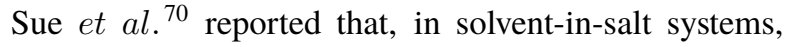

high viscosity and incomplete solvation shell facilitate higher $\mathrm{Li}^{+}$transference number $\left(t_{\mathrm{Li}^{+}}=J_{\mathrm{Li}^{+}} / J\right)$, which is unlike in conventional salt-in-solvent electrolytes. In our system, the $\mathrm{Li}^{+}$transference number does not increase with $\mathrm{Li}_{2} \mathrm{~S}_{x}$ concentration (see Fig. 6e). Instead, it stays around 0.2 for a wide range of PS concentrations without LiTFSI (as also demonstrated experimentally 6 ). The low value can be explained by the fact that $\mathrm{Li}^{+}$ions are mostly moving collectively together with PS ions. The constant value of the transference number is also in accordance with the weak dependence of coordination numbers on PS concentrations. Expectedly, with $0.5 \mathrm{M}$ LiTFSI $t_{\mathrm{Li}^{+}}$increases, since additional $\mathrm{Li}^{+}$that come from LiTFSI are not bound to PS and contribute to the conductivity to a greater extent. In the latter case, the contribution of PS to conductivity becomes negligible, as evident from the low PS transference number in the presence of LiTFSI (see Fig. S6a and b). Again, due to the strong binding between $\mathrm{Li}^{+}$and $\mathrm{S}_{4}{ }^{2-}, \mathrm{Li}_{2} \mathrm{~S}_{4}$ behaves as a neutral species and is therefore not subject to the electric field. We presume that these short PS chains are more likely to participate in the shuttle mechanism during the charge.

3.4. Diffusion coefficients. Evaluating the long-time mean square displacement, we now compute the self diffusion coefficients of all the species in our system. The diffusion coefficients from MD simulations are compared with measured values from PFG-NMR ${ }^{28}$ in Fig. $8 \mathrm{a}$, which suggests fair agreement. At this point, we remark that without the ECC treatment of ionic charges, the MD results would deviate from the experiments by an order of magnitude as we demonstrate in ESI (Fig. S7). Namely, without $\mathrm{ECC}$, the $\mathrm{PS}-\mathrm{Li}^{+}$binding is unrealistically strong and thus exaggerates the ion-pair formation. 71,74

Shorter PS chains $\left(\mathrm{S}_{4}{ }^{2-}\right)$ diffuse faster than longer ones (such as $\mathrm{S}_{6}{ }^{2-}$ and $\mathrm{S}_{8}{ }^{2-}$ ), which can simply be explained in terms of the Stokes-Einstein relations, where the diffusion coefficient is inversely proportional to the particle's effective size. We furthermore notice (Fig. 8 a) that the diffusion coefficients of $\mathrm{Li}^{+}$and $\mathrm{S}_{x}{ }^{2-}$ in the solvent (without TFSI ${ }^{-}$ions) are of the same order. This can be related to collective diffusion of $\mathrm{S}_{x}{ }^{2-}$ and $\mathrm{Li}^{+}$ ions due to their strong association. As we increase the PS concentration, the $\mathrm{Li}^{+}$diffusivity monotonically decreases (Fig. 8b), as also demonstrated experimentally ${ }^{67}$ This effect can be ascribed to an increasing viscosity in more concentrated PS solutions (Fig. S8a in ESI). At low concentrations of PS, the diffusion coefficient of $\mathrm{Li}^{+}$significantly depends on the PS type. Namely, shorter PS $\left(\mathrm{Li}_{2} \mathrm{~S}_{4}\right)$ promotes higher diffusion 
(a) $0.25 \mathrm{M} \mathrm{Li}_{2} \mathrm{~S}_{x}$ in DME:DOL

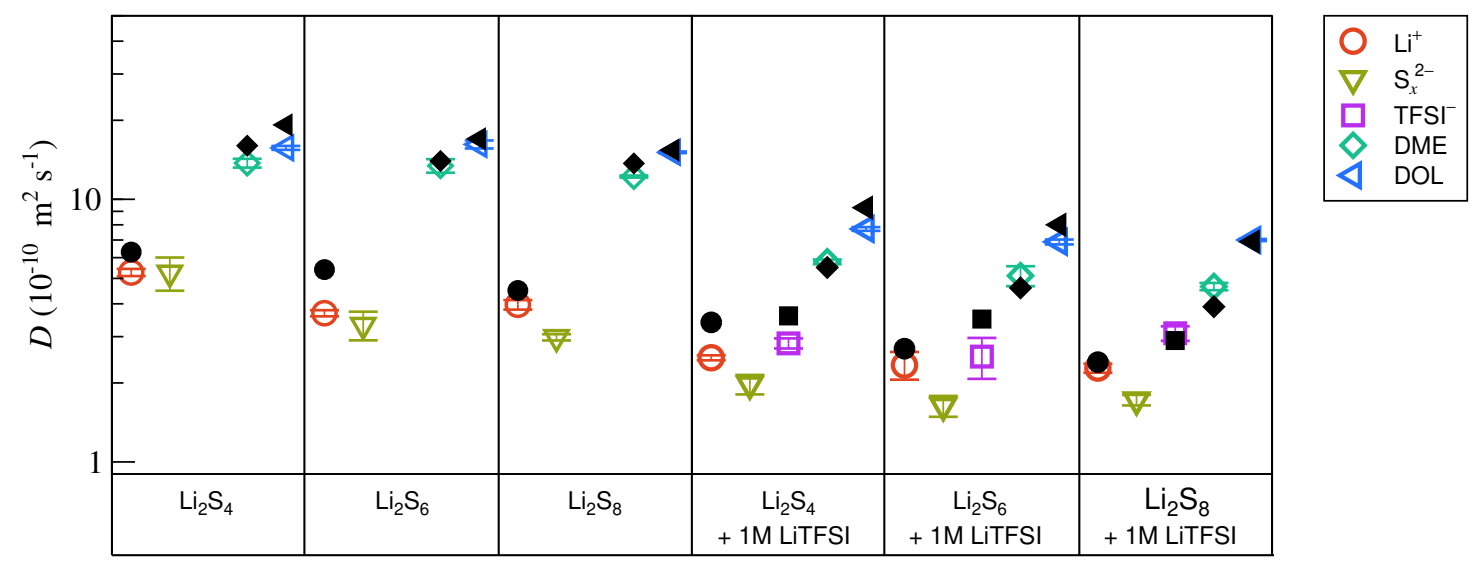

(b) $\mathrm{Li}_{2} \mathrm{~S}_{x}$

(c) $\mathrm{Li}_{2} \mathrm{~S}_{6}+\mathrm{LiTFSI}$

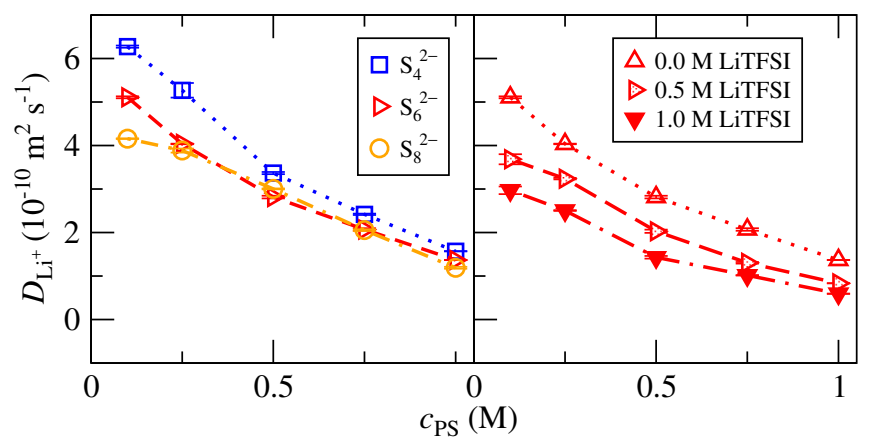

Figure 8: (a) Long-time self-diffusion coefficients $D$ of all species in the $0.25 \mathrm{M}$ of the polysulfide as obtained from MD simulations

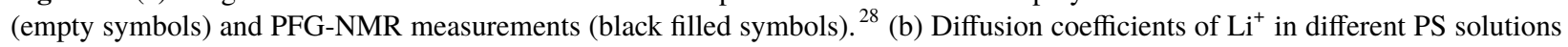
as a function of the PS concentration from MD simulations. (c) Diffusion coefficients of $\mathrm{Li}^{+}$as a function of $\mathrm{Li}_{2} \mathrm{~S}_{6}$ concentration in the presence of different amounts of LiTFSI from MD simulations.

than longer PS $\left(\mathrm{Li}_{2} \mathrm{~S}_{6}\right.$ and $\left.\mathrm{Li}_{2} \mathrm{~S}_{8}\right)$. Yet, this differences disappear at higher concentrations of PS (above around $0.5 \mathrm{M}$ ). Interestingly, adding $1 \mathrm{M}$ LiTFSI reduces the diffusion up to about $50 \%$ (Fig. $8 \mathrm{a}$, c), as also reported in a previous simulation study. $\frac{28}{.28}$ Also this effect can be attributed to an increased viscosity when LiTFSI is added (see Fig. S8b in ESI). The estimated viscosity of $\mathrm{Li}_{2} \mathrm{~S}_{6}$ in DME/DOL with 1M LiTFSI is about a factor of three higher than without LiTFSI. As we have seen, viscosity plays a critical role in ionic transport in the PS solutions. Introducing LiTFSI into dilute PS solutions increases the number of dissociated $\mathrm{Li}^{+}$and leads to a higher conductivity (Fig. 6. Yet, this ionic effect fades out compared to an increasing viscosity at high PS concentrations.
3.5. Clustering. Due to the high attraction between terminal sulfur ends of PS and $\mathrm{Li}^{+}$ions, occasionally two different PS ions can bind to the same $\mathrm{Li}^{+}$ion, such that the $\mathrm{Li}^{+}$ion represents a "bridging" element for the two PS ions. This can result into supramolecular clusters that are composed of several PS chains and $\mathrm{Li}^{+}$ions. Figure 9 shows the cluster-size distribution $P(N)$ of PS in $\log$-lin presentation. As seen, the clusters do not have a characteristic size but are extremely polydisperse and roughly follow an exponential distribution $P(N) \sim$ $\exp (-N / \bar{N})$, where $\bar{N}$ is the mean size of the clusters. ${ }^{75}$ Some deviations from the exponential behavior occur for larger clusters at higher concentrations, suggesting a cooperative binding. Yet, the cluster size distribution indicates that single monomers and small PS clusters prevail.

Clearly, higher concentrations of PS increase the proportion of larger clusters, simply because the probability of 


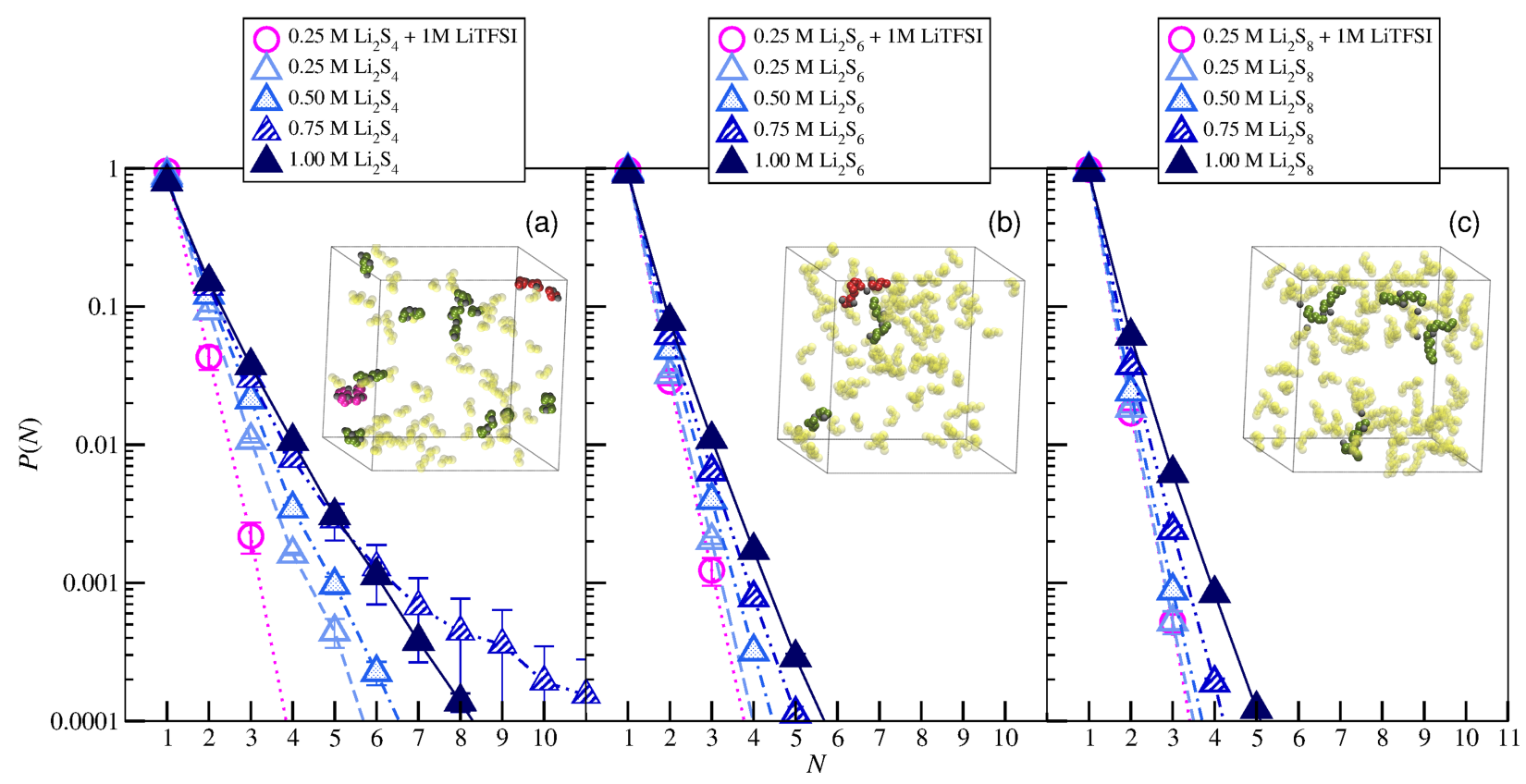

Figure 9: Cluster size distributions for (a) $\mathrm{Li}_{2} \mathrm{~S}_{4}$, (b) $\mathrm{Li}_{2} \mathrm{~S}_{6}$, and (c) $\mathrm{Li}_{2} \mathrm{~S}_{8}$ in DME:DOL. Insets show representative simulation snapshots of $0.25 \mathrm{M}$ of the polysulfide. Clusters of different sizes are shown in different colors: yellow $(N=1)$, green $(N=2)$, pink $(N=3)$, and red $(N=4)$.

different PS chains to meet is higher in a more concentrated solution. Even higher concentrations, approaching the solubility limit (not shown), thus provide critical nucleation sites for formation of large cluster precipitates. The morphology of the cluster may thus depend on the growth and nucleation rates. .69

Moreover, comparing the clustering of different PS lengths reveals that the shorter PSs tend to form larger clusters more readily, especially at high concentrations, compared with longer PSs. A higher frequency of clusters up to 80 atoms was also observed recently for the shorter chains $\left(\mathrm{S}_{4}^{2-}\right)$ when compared to the longer ones. ${ }^{35}$ The reason lies in the stronger charge localization at $\mathrm{S}$ termini in shorter PS chains, as already discussed above. These results are in line with Vijayakumar et al. ${ }^{[24}$ who reported that $\mathrm{Li}_{2} \mathrm{~S}_{4}$ favors dimer formation, whereas $\mathrm{Li}_{2} \mathrm{~S}_{8}$ favors monomer formation in dimethyl sulfoxide (DMSO) solvent, while $\mathrm{Li}_{2} \mathrm{~S}_{6}$ being somewhere in between. Representative simulation snapshots are shown in the insets of Fig. 9, featuring larger cluster formation in the case of $\mathrm{Li}_{2} \mathrm{~S}_{4}$ than in the other two cases. Note that even shorter PS chains, like $\mathrm{S}_{2}{ }^{2-}$, are even less soluble in the existing solvent and tend to precipitate out of solution even at very low concentrations ${ }^{2361176}$ (see Figs. S9 in ESI for the analysis of $\mathrm{S}_{2}{ }^{2-}$ and Fig. S10 for snapshot). Interestingly, adding $1 \mathrm{M}$ of LiTFSI inhibits the cluster- ing for $\mathrm{Li}_{2} \mathrm{~S}_{4}$ (Figs. 9 a-c), as also reported before. ${ }^{28229}$ This can, however, not be claimed for the other two PS species. As discussed above, the presence of LiTFSI does not significantly influence the $\mathrm{Li}^{+}-\mathrm{PS}$ binding as seen from coordination numbers of $S_{\text {ter }}$. However, it apparently tends to inhibit cluster formation via other mechanisms, such as increased electrostatic screening due to LiTFSI ions. This influence of the ionic strength on the Li-PS network also impacts the shuttle effect in $\mathrm{Li} / \mathrm{S}$ batteries. ${ }^{26177}$ Sustaining the Li-S networks by using low ion-pairing salt, can decrease the shuttle effect and increase the cycle performance of the batteries. $\frac{78779}{}$

\section{CONCLUSIONS}

We developed an atomistic model for polysulfides (PS) in an organic functional solvent that is currently in use for the development of $\mathrm{Li} / \mathrm{S}$ batteries. We focused particularly on structural and dynamic properties of three different sizes of PS ions, $\mathrm{S}_{4}{ }^{2-}, \mathrm{S}_{6}{ }^{2-}, \mathrm{S}_{8}{ }^{2-}$ in the presence of $\mathrm{Li}^{+}$and $\mathrm{TFSI}^{-}$ions. The conductivity and diffusion coefficients of PS solutions are validated by experimental measurements. Conductivities of PS solutions first exhibit an increase with the $\mathrm{Li}_{2} \mathrm{~S}_{x}$ concentrations and eventually a saturation at around $0.5 \mathrm{M}$. The saturation in the conductivity can be linked to viscosity and 
the ionic correlations between PS and $\mathrm{Li}^{+}$, which also lead to occurrence of supramolecular clusters. The tendency of clustering increases with the concentration of $\mathrm{Li}_{2} \mathrm{~S}_{x}$ and is more pronounced for shorter PS ions (i.e., $x=4$ ). Shorter chains have their electronic density more strongly localized at the terminal sulfur atoms than longer chains, thus facilitating the electrostatic attraction with $\mathrm{Li}^{+}$.

The addition of $\mathrm{TFSI}^{-}$ions leads to larger amount of dissociated $\mathrm{Li}^{+}$ions and to a noticeable increase in the viscosity. The dissociated $\mathrm{Li}^{+}$contributes to the conductivity considerably, on the other hand, increased viscosity inhibits the conductivity at larger PS concentrations. The presence of LiTFSI also reduces cluster formation of shorter PS ions. Even though TFSI ions do not significantly reduce the $\mathrm{Li}^{+}-\mathrm{PS}$ binding, they weaken the binding between multiple PS ions into clusters, partially because of a higher ionic strength. Thus, LiTFSI increases the solubility of PS ions and with that enhances the shuttle effect in the Li/S batteries.

Our simulation results of PS solutions reveal that structural and transport properties are subject to subtle interactions among ions and solvents molecules, which should be considered carefully when it comes to the design of electrolytes for Li/S batteries. $\frac{80}{}$ In the next step, studies on different types of anions in Li/PS solutions 81 as well as in confining electrode materials are envisioned.

\section{CONFLICT OF INTEREST}

There are no conflicts of interest to declare.

Supporting Information Description. Force field development for Sulfur non-bonded parameters; Conductivity; Lennard-Jones parameterization based on conductivity; Diffusion coefficients: finite size correction (FSC) ( $\mathrm{Li}_{2} \mathrm{~S}_{4}$ in DME:DOL); Cluster analysis; Radial distribution function (RDF): $0.25 \mathrm{M} \mathrm{Li}_{2} \mathrm{~S}_{x}$ in DME:DOL; The integral of the correlation function; Transference number; Diffusion coefficients without ECC; Viscosity; Cluster analysis and snapshot for $\mathrm{Li}_{2} \mathrm{~S}_{2}$ in DME:DOL.

\section{ACKNOWLEDGMENTS}

The authors thank Richard Chudoba for useful discussions. M.K. acknowledges the financial support from the Slovenian Research Agency (research core funding no. P1-0055).

\section{References}

(1) Fang, R.; Zhao, S.; Sun, Z.; Wang, D.-W.; Cheng, H.-M.; Li, F. More reliable lithium-sulfur batteries: status, solutions and prospects. $A d v$. Mater. 2017, 29, 1606823.

(2) Kang, W.; Deng, N.; Ju, J.; Li, Q.; Wu, D.; Ma, X.; Li, L.; Naebe, M.; Cheng, B. A review of recent developments in rechargeable lithium-sulfur batteries. Nanoscale 2016, $8,16541-16588$.

(3) Schipper, F.; Aurbach, D. A brief review: Past, present and future of lithium ion batteries. Russ. J. Electrochem. 2016, 52, 1095-1121.

(4) Wild, M.; O’Neill, L.; Zhang, T.; Purkayastha, R.; Minton, G.; Marinescu, M.; Offer, G. Lithium sulfur batteries, a mechanistic review. Energy Environ. Sci. 2015, 8, 3477-3494.

(5) Manthiram, A.; Fu, Y.; Chung, S.-H.; Zu, C.; $\mathrm{Su}$, Y.-S. Rechargeable lithium-sulfur batteries. Chem. Rev. 2014, 114, 11751-11787.

(6) Cuisinier, M.; Cabelguen, P.-E.; Evers, S.; He, G.; Kolbeck, M.; Garsuch, A.; Bolin, T.; Balasubramanian, M.; Nazar, L. F. Sulfur speciation in Li-S batteries determined by operando X-ray absorption spectroscopy. J. Phys. Chem. Lett. 2013, 4, 32273232.

(7) Dominko, R.; Patel, M. U.; Lapornik, V.; Vizintin, A.; Koželj, M.; N. Tušar, N.; Arčon, I.; Stievano, L.; Aquilanti, G. Analytical detection of polysulfides in the presence of adsorption additives by operando x-ray absorption spectroscopy. J. Phys. Chem. C 2015, 119, 19001-19010.

(8) Busche, M. R.; Adelhelm, P.; Sommer, H.; Schneider, H.; Leitner, K.; Janek, J. Systematical electrochemical study on the parasitic shuttle-effect in lithium-sulfur-cells at different temperatures and different rates. J. Power Sources 2014, 259, 289299.

(9) Mikhaylik, Y. V.; Akridge, J. R. Polysulfide shuttle study in the Li/S battery system. J. Electrochem. Soc. 2004, 151, A1969-A1976.

(10) Cleaver, T.; Kovacik, P.; Marinescu, M.; Zhang, T.; Offer, G. Perspective-commercializing lithium sulfur batteries: Are we doing the right research? J. Electrochem. Soc. 2018, 165, A6029-A6033.

(11) Ji, X.; Nazar, L. F. Advances in Li-S batteries. J. Mater. Chem. 2010, 20, 9821-9826.

(12) Wang, D.-W.; Zhou, G.; Li, F.; Wu, K.-H.; Lu, G. Q. M.; Cheng, H.-M.; Gentle, I. R. A microporousmesoporous carbon with graphitic structure for a high-rate stable sulfur cathode in carbonate 
solvent-based Li-S batteries. Phys. Chem. Chem. Phys. 2012, 14, 8703-8710.

(13) Zhang, B.; Qin, X.; Li, G.; Gao, X. Enhancement of long stability of sulfur cathode by encapsulating sulfur into micropores of carbon spheres. Energy Environ. Sci. 2010, 3, 1531-1537.

(14) Su, Y.-S.; Manthiram, A. Lithium-sulphur batteries with a microporous carbon paper as a bifunctional interlayer. Nat. Commun. 2012, 3, 1166.

(15) Li, G.; Wang, S.; Zhang, Y.; Li, M.; Chen, Z.; Lu, J. Revisiting the role of polysulfides in lithium-sulfur batteries. Adv. Mater. 2018, 1705590.

(16) Lee, C.-W.; Pang, Q.; Ha, S.; Cheng, L.; Han, S.D.; Zavadil, K. R.; Gallagher, K. G.; Nazar, L. F.; Balasubramanian, M. Directing the lithium-sulfur reaction pathway via sparingly solvating electrolytes for high energy density batteries. ACS Cent. Sci. 2017, 3, 605-613.

(17) Scheers, J.; Fantini, S.; Johansson, P. A review of electrolytes for lithium-sulphur batteries. J. Power Sources 2014, 255, 204-218.

(18) Chen, S.; Wang, D.; Zhao, Y.; Wang, D. Superior performance of a lithium-sulfur battery enabled by a dimethyl trisulfide containing electrolyte. Small Methods 2018, 2, 1800038.

(19) Urbonaite, S.; Novák, P. Importance of 'unimportant' experimental parameters in Li-S battery development. J. Power Sources 2014, 249, 497-502.

(20) Fan, F. Y.; Pan, M. S.; Lau, K. C.; Assary, R. S.; Woodford, W. H.; Curtiss, L. A.; Carter, W. C.; Chiang, Y.-M. Solvent effects on polysulfide redox kinetics and ionic conductivity in lithium-sulfur batteries. J. Electrochem. Soc. 2016, 163, A3111A3116.

(21) Wang, B.; Alhassan, S. M.; Pantelides, S. T. Formation of large polysulfide complexes during the lithium-sulfur battery discharge. Phys. Rev. Appl. 2014, 2, 034004.

(22) Partovi-Azar, P.; Kühne, T. D.; Kaghazchi, P. Evidence for the existence of Li2S2 clusters in lithiumsulfur batteries: ab initio Raman spectroscopy simulation. Phys. Chem. Chem. Phys. 2015, 17, 22009-22014.

(23) Pascal, T. A.; Wujcik, K. H.; Wang, D. R.; Balsara, N. P.; Prendergast, D. Thermodynamic origins of the solvent-dependent stability of lithium polysulfides from first principles. Phys. Chem. Chem. Phys. 2017, 19, 1441-1448.

(24) Vijayakumar, M.; Govind, N.; Walter, E.; Burton, S. D.; Shukla, A.; Devaraj, A.; Xiao, J.; Liu, J.; Wang, C.; et al, Molecular structure and stability of dissolved lithium polysulfide species. Phys. Chem. Chem. Phys. 2014, 16, 10923-10932.

(25) Yu, T.; Li, F.; Liu, C.; Zhang, S.; Xu, H.; Yang, G. Understanding the role of lithium sulfide clusters in lithium-sulfur batteries. J. Mater. Chem. A 2017, 5, 9293-9298.

(26) Kamphaus, E. P.; Balbuena, P. B. First-principles investigation of lithium polysulfide structure and behavior in solution. J. Phys. Chem. C 2017, 121, 21105-21117.

(27) Liu, Z.; Deng, H.; Hu, W.; Gao, F.; Zhang, S.; Balbuena, P. B.; Mukherjee, P. P. Revealing reaction mechanisms of nanoconfined Li $2 \mathrm{~S}$ : implications for lithium-sulfur batteries. Phys. Chem. Chem. Phys. 2018, 20, 11713-11721.

(28) Rajput, N. N.; Murugesan, V.; Shin, Y.; Han, K. S.; Lau, K. C.; Chen, J.; Liu, J.; Curtiss, L. A.; Mueller, K. T.; Persson, K. A. Elucidating the solvation structure and dynamics of lithium polysulfides resulting from competitive salt and solvent interactions. Chem. Mater. 2017, 29, 3375-3379.

(29) Osella, S.; Minoia, A.; Quarti, C.; Cornil, J.; Lazzaroni, R.; Goffin, A.-L.; Guillaume, M.; Beljonne, D. Modelling coupled ion motion in electrolyte solutions for lithium-sulfur batteries. Batteries \& Supercaps

(30) Park, J.-W.; Yamauchi, K.; Takashima, E.; Tachikawa, N.; Ueno, K.; Dokko, K.; Watanabe, M. Solvent effect of room temperature ionic liquids on electrochemical reactions in lithium-sulfur batteries. J. Phys. Chem. C 2013, 117, 4431-4440.

(31) Zhang, C.; Yamazaki, A.; Murai, J.; Park, J.W.; Mandai, T.; Ueno, K.; Dokko, K.; Watanabe, M. Chelate effects in glyme/lithium bis (trifluoromethanesulfonyl) amide solvate ionic liquids, part 2: importance of solvate-structure stability for electrolytes of lithium batteries. J. Phys. Chem. C 2014, 118, 17362-17373.

(32) Pan, H.; Wei, X.; Henderson, W. A.; Shao, Y.; Chen, J.; Bhattacharya, P.; Xiao, J.; Liu, J. On the way toward understanding solution chemistry of lithium polysulfides for high energy $\mathrm{Li}-\mathrm{S}$ redox flow batteries. Adv. Energy Mater. 2015, 5, 1500113.

(33) Zheng, D.; Zhang, X.; Li, C.; McKinnon, M. E.; Sadok, R. G.; Qu, D.; Yu, X.; Lee, H.-S.; Yang, X.Q.; Qu, D. Quantitative chromatographic determination of dissolved elemental sulfur in the nonaqueous electrolyte for lithium-sulfur batteries. $J$. Electrochem. Soc. 2015, 162, A203-A206.

(34) Yu, S.-H.; Huang, X.; Schwarz, K.; Huang, R.; 
Arias, T. A.; Brock, J. D.; Abruña, H. D. Direct visualization of sulfur cathodes: new insights into Li-S batteries via operando X-ray based methods. Energy Environ. Sci. 2018, 11, 202-210.

(35) Andersen, A.; Rajput, N. N.; Han, K. S.; Pan, H.; Govind, N.; Persson, K. A.; Mueller, K. T.; Murugesan, V. Structure and dynamics of polysulfide clusters in a nonaqueous solvent mixture of 1, 3dioxolane and 1,2-dimethoxyethane. Chem. Mater. 2019 ,

(36) Park, C.; Kanduč, M.; Chudoba, R.; Ronneburg, A.; Risse, S.; Ballauff, M.; Dzubiella, J. Molecular simulations of electrolyte structure and dynamics in lithium-sulfur battery solvents. $J$. Power Sources 2018, 373, 70-78.

(37) Jorgensen, W. L.; Maxwell, D. S.; Tirado-Rives, J. Development and testing of the OPLS all-atom force field on conformational energetics and properties of organic liquids. J. Am. Chem. Soc 1996, 118, 11225-11236.

(38) Anderson, P. M.; Wilson, M. R. Developing a force field for simulation of poly (ethylene oxide) based upon ab initio calculations of 1, 2dimethoxyethane. Mol. Phys. 2005, 103, 89-97.

(39) Canongia Lopes, J. N.; Pádua, A. A. Molecular force field for ionic liquids composed of triflate or bistriflylimide anions. J. Phys. Chem. B 2004, 108, 16893-16898.

(40) Dang, L. X. Development of nonadditive intermolecular potentials using molecular dynamics: solvation of $\mathrm{Li}^{+}$and $\mathrm{F}^{-}$ions in polarizable water. J. Chem. Phys. 1992, 96, 6970-6977.

(41) Leontyev, I.; Vener, M.; Rostov, I.; Basilevsky, M.; Newton, M. D. Continuum level treatment of electronic polarization in the framework of molecular simulations of solvation effects. J. Chem. Phys. 2003, 119, 8024-8037.

(42) Leontyev, I.; Stuchebrukhov, A. Electronic continuum model for molecular dynamics simulations. $J$. Chem. Phys. 2009, 130, 085102.

(43) Leontyev, I.; Stuchebrukhov, A. Electronic polarizability and the effective pair potentials of water. J. Chem. Theory Comput. 2010, 6, 3153-3161.

(44) Leontyev, I.; Stuchebrukhov, A. Electronic continuum model for molecular dynamics simulations of biological molecules. J. Chem. Theory Comput. 2010, 6, 1498-1508.

(45) Leontyev, I.; Stuchebrukhov, A. Accounting for electronic polarization in non-polarizable force fields. Phys. Chem. Chem. Phys. 2011, 13, 26132626.
(46) Leontyev, I. V.; Stuchebrukhov, A. A. Polarizable mean-field model of water for biological simulations with AMBER and CHARMM force fields. $J$. Chem. Theory Comput. 2012, 8, 3207-3216.

(47) Leontyev, I. V.; Stuchebrukhov, A. A. Polarizable molecular interactions in condensed phase and their equivalent nonpolarizable models. J. Chem. Phys. 2014, 141, 014103.

(48) Frisch, M.; Trucks, G.; Schlegel, H.; Scuseria, G.; Robb, M.; Cheeseman, J.; Scalmani, G.; Barone, V.; Mennucci, B.; Petersson, G. et al. Official Gaussian 09 literature citation. 2014.

(49) Sorin, E. J.; Pande, V. S. Exploring the helix-coil transition via all-atom equilibrium ensemble simulations. Biophys. J. 2005, 88, 2472-2493.

(50) MacKerell Jr, A. D.; Bashford, D.; Bellott, M.; Dunbrack Jr, R. L.; Evanseck, J. D.; Field, M. J.; Fischer, S.; Gao, J.; Guo, H.; et al, All-atom empirical potential for molecular modeling and dynamics studies of proteins. J. Phys. Chem. B 1998, 102, 3586-3616.

(51) Levitt, M.; Hirshberg, M.; Sharon, R.; Daggett, V. Potential energy function and parameters for simulations of the molecular dynamics of proteins and nucleic acids in solution. Comput. Phys. Commun. 1995, 91, 215-231.

(52) Nemethy, G.; Pottle, M. S.; Scheraga, H. A. Energy parameters in polypeptides. 9. Updating of geometrical parameters, nonbonded interactions, and hydrogen bond interactions for the naturally occurring amino acids. J. Phys. Chem. 1983, 87, 1883-1887.

(53) Rappé, A. K.; Casewit, C. J.; Colwell, K.; Goddard Iii, W.; Skiff, W. UFF, a full periodic table force field for molecular mechanics and molecular dynamics simulations. J. Am. Chem. Soc 1992, 114, 10024-10035.

(54) Mayo, S. L.; Olafson, B. D.; Goddard, W. A. DREIDING: a generic force field for molecular simulations. J. Phys. Chem. 1990, 94, 8897-8909.

(55) Abraham, M.; Van Der Spoel, D.; Lindahl, E.; Hess, B. GROMACS development team, GROMACS user manual version 5.1. 4. 2016.

(56) Martínez, L.; Andrade, R.; Birgin, E. G.; Martínez, J. M. PACKMOL: a package for building initial configurations for molecular dynamics simulations. J. Comput. Chem. 2009, 30, 2157-2164.

(57) Berendsen, H. J.; Postma, J. v.; van Gunsteren, W. F.; DiNola, A.; Haak, J. Molecular dynamics with coupling to an external bath. J. Chem. Phys. 1984, 81, 3684-3690. 
(58) Dommert, F.; Schmidt, J.; Qiao, B.; Zhao, Y.; Krekeler, C.; Delle Site, L.; Berger, R.; Holm, C. A comparative study of two classical force fields on statics and dynamics of $[\mathrm{EMIM}]\left[\mathrm{BF}_{4}\right]$ investigated via molecular dynamics simulations. $J$. Chem. Phys. 2008, 129, 224501.

(59) Neumann, M. Dipole moment fluctuation formulas in computer simulations of polar systems. Mol. Phys 1983, 50, 841-858.

(60) Hansen, J.-P.; McDonald, I. R. Theory of simple liquids; Elsevier, 1990.

(61) Barchasz, C.; Molton, F.; Duboc, C.; Leprêtre, J.C.; Patoux, S.; Alloin, F. Lithium/sulfur cell discharge mechanism: an original approach for intermediate species identification. Anal. Chem. 2012, 84, 3973-3980.

(62) Ben-Yaakov, D.; Andelman, D.; Podgornik, R. Dielectric decrement as a source of ion-specific effects. J. Chem. Phys. 2011, 134, 074705.

(63) Glueckauf, E. Bulk dielectric constant of aqueous electrolyte solutions. J. Chem. Soc. Faraday Trans. 1964, 60, 1637-1645.

(64) Wei, Y.-Z.; Chiang, P.; Sridhar, S. Ion size effects on the dynamic and static dielectric properties of aqueous alkali solutions. J. Chem. Phys. 1992, 96, 4569-4573.

(65) Chandra, A. Static dielectric constant of aqueous electrolyte solutions: Is there any dynamic contribution? J. Chem. Phys. 2000, 113, 903-905.

(66) Han, K. S.; Chen, J.; Cao, R.; Rajput, N. N.; Murugesan, V.; Shi, L.; Pan, H.; et al, Effects of anion mobility on electrochemical behaviors of lithiumsulfur batteries. Chem. Mater. 2017, 29, 9023 9029.

(67) Safari, M.; Kwok, C. Y.; Nazar, L. F. Transport properties of polysulfide species in lithium-sulfur battery electrolytes: coupling of experiment and theory. ACS Cent. Sci. 2016, 2, 560-568.

(68) Altenberger, A.; Friedman, H. L. Theory of conductance and related isothermal transport coefficients in electrolytes. J. Chem. Phys. 1983, 78, 4162-4173.

(69) Zheng, J.; Lv, D.; Gu, M.; Wang, C.; Zhang, J.-G.; Liu, J.; Xiao, J. How to obtain reproducible results for lithium sulfur batteries? J. Electrochem. Soc. 2013, 160, A2288-A2292.

(70) Suo, L.; Hu, Y.-S.; Li, H.; Armand, M.; Chen, L. A new class of solvent-in-salt electrolyte for highenergy rechargeable metallic lithium batteries. Nat. Commun. 2013, 4, 1481.

(71) Pluhařová, E.; Mason, P. E.; Jungwirth, P. Ion pair- ing in aqueous lithium salt solutions with monovalent and divalent counter-anions. J. Phys. Chem. A 2013, 117, 11766-11773.

(72) Vazdar, M.; Jungwirth, P.; Mason, P. E. Aqueous guanidinium-carbonate interactions by molecular dynamics and neutron scattering: relevance to ionprotein interactions. J. Phys. Chem. B 2013, 117, 1844-1848.

(73) Pegado, L.; Marsalek, O.; Jungwirth, P.; Wernersson, E. Solvation and ion-pairing properties of the aqueous sulfate anion: explicit versus effective electronic polarization. Phys. Chem. Chem. Phys. 2012, 14, 10248-10257.

(74) Mason, P. E.; Wernersson, E.; Jungwirth, P. Accurate description of aqueous carbonate ions: An effective polarization model verified by neutron scattering. J. Phys. Chem. B 2012, 116, 8145-8153.

(75) Israelachvili, J. N. Intermolecular and surface forces; Academic press, 2011.

(76) Ding, N.; Li, X.; Chien, S. W.; Liu, Z.; Zong, Y. In situ monitoring the viscosity change of an electrolyte in a Li-S battery. ChemComm 2017, 53, 10152-10155.

(77) Peled, E.; Sternberg, Y.; Gorenshtein, A.; Lavi, Y. Lithium-sulfur battery: evaluation of dioxolanebased electrolytes. J. Electrochem. Soc. 1989, 136, 1621-1625.

(78) Shyamsunder, A.; Beichel, W.; Klose, P.; Pang, Q.; Scherer, H.; Hoffmann, A.; Murphy, G. K.; Krossing, I.; Nazar, L. F. Inhibiting polysulfide shuttle in lithium-sulfur batteries through low-ion-pairing salts and a triflamide solvent. Angew. Chem. 2017, 56, 6192-6197.

(79) Chen, J.; Han, K. S.; Henderson, W. A.; Lau, K. C.; Vijayakumar, M.; Dzwiniel, T.; Pan, H.; Curtiss, L. A.; Xiao, J.; et al, Restricting the solubility of polysulfides in Li-S batteries via electrolyte salt selection. Adv. Energy Mater. 2016, 6, 1600160.

(80) Chaudhari, M. I.; Muralidharan, A.; Pratt, L. R.; Rempe, S. B. Assessment of simple models for molecular simulation of ethylene carbonate and propylene carbonate as solvents for electrolyte solutions. Top. Curr. Chem. 2018, 376, 7.

(81) Lesch, V.; Jeremias, S.; Moretti, A.; Passerini, S.; Heuer, A.; Borodin, O. A combined theoretical and experimental study of the influence of different anion ratios on lithium ion dynamics in ionic liquids. J. Phys. Chem. B 2014, 118, 7367-7375. 